\title{
MEMS for pico- to micro-satellites
}

\author{
H.R. Shea* \\ Microsystems for Space Technologies Laboratory (LMTS) \\ EPFL, Ecole Polytechnique Fédérale de Lausanne, \\ Rue Jaquet Droz 1, CP 526, CH-2002 Neuchâtel, Switzerland
}

\begin{abstract}
MEMS sensors, actuators, and sub-systems can enable an important reduction in the size and mass of spacecrafts, first by replacing larger and heavier components, then by replacing entire subsystems, and finally by enabling the microfabrication of highly integrated picosats. Very small satellites (1 to $100 \mathrm{~kg}$ ) stand to benefit the most from MEMS technologies. These small satellites are typically used for science or technology demonstration missions, with higher risk tolerance than multi-ton telecommunication satellites. While MEMS are playing a growing role on Earth in safetycritical applications, in the harsh and remote environment of space, reliability is still the crucial issue, and the absence of an accepted qualification methodology is holding back MEMS from wider use. An overview is given of the range of MEMS applications in space. An effective way to prove that MEMS can operate reliably in space is to use them in space: we illustrate how Cubesats (1 kg, 1 liter, cubic satellites in a standardized format to reduce launch costs) can serve as low-cost vectors for MEMS technology demonstration in space. The Cubesat SwissCube developed in Switzerland is used as one example of a rapid way to fly new microtechnologies, and also as an example of a spacecraft whose performance is only possible thanks to MEMS.
\end{abstract}

Keywords: MEMS, spacecraft, satellites, nanosatellites, cubesats, space

\section{OVERVIEW OF MEMS APPLICATIONS IN SPACE}

In 2008 the market for MEMS (MicroElectroMechanical Systems) devices was nearly $\$ 8$ Billion according to Yole Développement [1]. MEMS encompass an enormous range of applications, from RF switching to projection display to inertial sensors to implantable pumps. Combining low mass, low power consumption, small volume and possible integration with control and sense electronics, MEMS seem ideal for space applications, where it costs approximately $10 ’ 000 \$$ to place one $\mathrm{kg}$ in low Earth orbit (LEO).

Reliability is a key concern for spacecraft, in view of the very larger development costs, near impossibility of repair (the service missions to the Hubble Space Telescope are the notable exception), and limited launch slots. One major difference between operation on Earth and in Space is the radiation level [2]. Other space-specific reliability concerns are thermal cycling and thermal shocks, vibration and mechanical shock at launch and stage/heat shield separation, and operation in very high vacuum. The typical service life of a telecommunication satellite is 15 years, during which time the spacecraft must operate continuously and flawlessly. For this reason, and because of the need for radiation tolerance, new technologies are generally accepted in space applications only many years later than in consumer electronics, except for cases where a new technology is required for a mission, or allows dramatic performance enhancement or mass reduction. This was the case for instance for FPGAs and is now the case for MEMS.

The fraction of the $\$ 8$ Billion MEMS market for space is very small, and MEMS for use in space often have unique requirements. MEMS have been proposed for a number of space applications, as lighter and smaller replacement parts or as entire new systems [3][4][5], or as a means to provide affordable redundancy and hence improved operational reliability [6]. Few MEMS components have been flown in space. MEMS for space are currently either commercial offthe-shelf (COTS) parts such as accelerometers or gyroscopes that have been subjected to additional testing and qualification, or new MEMS devices that are crucial enabling technologies for science missions which cannot proceed without MEMS (such as the AFM on the Phoenix mission on Mars [7]). In the medium term MEMS technology will allow sub-systems (such as attitude determination, phased array antennas, Earth sensors, optical switches, low-thrust

*herbert.shea@epfl.ch ; http://lmts.epfl.ch

MOEMS and Miniaturized Systems VIII, edited by David L. Dickensheets, Harald Schenk, Wibool Piyawattanametha Proc. of SPIE Vol. 7208, 72080M · @ 2009 SPIE · CCC code: 0277-786X/09/\$18 · doi: 10.1117/12.810997 
propulsion systems) to be reduced significantly in size and mass. In the longer run MEMS can enable new classes of extremely small, intelligent, self managing and relatively low-cost batch-produced picosatellites operating in constellations. Most MEMS devices currently under development for specific space applications are not intended for picosallites, principally because most satellites currently have masses larger than $100 \mathrm{~kg}$. However pico and nanosatellites are the main users in space of COTS MEMS parts (pico and nanosats are also the main users in space of COTS microcontrollers, batteries, etc since the development budget of these small satellites often precludes using standard space grade components). Some of the more developed MEMS areas for space are listed below, and can also be found in [8].

Inertial Navigation: accelerometers and gyroscopes are the most mature MEMS devices used in space, used to instrument launchers as well as rovers on Mars. Since these devices are available as COTS parts for commercial and military applications, it is likely that they can be re-qualified for space by de-rating (for one de-rating procedure see ref [14]). There have been several studies addressing COTS accelerometers for space [11][12][13]. COTS accelerometers have been shown to survive 1000 temperature cycles from $-65^{\circ} \mathrm{C}$ to $+150^{\circ} \mathrm{C}$, as well as 30,000 mechanical shocks of $2,000 \mathrm{G}$. To measure microgravity on the GeneSat1 mission, model 1221 capacitive micromachined accelerometers from Silicon Designs Inc., Issaquah, WA, USA were flown in 2006 in low Earth orbit [15].

RF Switches and Variable Capacitors: MEMS technologies enable the fabrication of very compact low-loss RF switches, as well as capacitors with a large tuning range. Such devices are just starting to be commercially available, for example from Radant MEMS [16] (Stow, MA, USA). RF switches were flown in space on the OPAL Picosats in 2000. They were stored in orbit for a year, and then successfully operated [17]. IMEC, in Leuven, Belgium, have led several studies into the reliability of RF MEMS for Space [18].

Atomic Force Microscope: an AFM was one of the scientific instruments on the 2008 Phoenix mission to Mars [7][19]. The piezoresistive single crystal silicon based AFM operated flawlessly on Mars. The scanner and cantilevers of the AFM were not radiation hardened but its control electronics were. This is a good example of rapid acceptance of MEMS in space. The near-impossibility of making an AFM without MEMS technology, and the fact that a failure of the AFM would not compromise the mission, allowed MEMS technology to be much more readily accepted.

Bio and Microfluidics: SU8 and other polymers, as well as Pyrex and silica can be used to fabricate micro-fluidic devices, including channels, nozzles and pumps. Such devices are principally being developed for medical or pharmaceutical (lab-on-a-chip) applications, and have been flow on the $25 \mathrm{~cm}$ long GeneSat 1 in 2006 to study the development of E. Coli cells in microgravity [15] [31] as well as in a small micro-reactor for yeast cell studies on the Space Shuttle and could also eventually be used as part of a propulsion system.

Bolometers: micromachining allows thermal isolation of small detectors, enabling both uncooled and cooled bolometer arrays to offer very high performance. Such devices are commercially available for earth-based IR detector applications. A MEMS bolometer from JPL/Caltech [10] is planned for the ESA/NASA Planck mission in 4-2009 to image the anisotropies of the Cosmic Background Radiation Field.

Optical instrumentation: the NIR-Spec (Near InfraRed Spectrometer) on the James Webb Space Telescope (JWST) will use MEMS-based micro-shutters manufactured by NASA/GSFC, whose radiation testing at $60 \mathrm{~K}$ is reported in [20]. The JWST is expected to be launched in 2013. The MEMS-based microshutter allows important performance improvement and several orders of magnitude mass reduction compared to a conventionally mechanical shutter array.

Optical Switching and Communication: The boom in optical MEMS between 1999 and 2002 led to the development of large optical switch matrices based on MEMS devices. There are ongoing European Space Agency (ESA) projects on the development and qualification methodology of such MEMS switches for space. There have been studies of COTS parts [21], but no devices have been flown.

Louvers for thermal control: MEMS louvers were developed by the Johns Hopkins University and NASA using a polysilicon process, allowing the emittance of the spacecraft to be varied [22]. Launched in 2006 aboard NASA's Space Technology 5 (ST5), they successfully operated as predicted.

Propulsion: MEMS propulsion systems produce low thrust, from $\mu \mathrm{N}$ to at most $1 \mathrm{~N}$. Such thrust levels can allow orbit maneuvers for small satellites, or attitude control and station keeping for larger satellites. There are several approaches to using MEMS for propulsion in space, reflecting the different propulsion technologies such as cold gas, hot gas, and electric propulsion. Electric propulsion allows reducing the amount of propellant required due to the high propulsion 
efficiency, but requires enough solar power to extract and accelerate the ions, often more power than available on very small satellites.

One approach to miniaturizing an ion-thruster is to micromachine and integrate the electron source, gas handling, nozzle and other components, is reported in [23]. Another approach to integrated MEMS colloid thrusters is described in [24][25][26], where arrays of micromachined ion sources using ionic liquids as fuel are used to create highly modulable thrust. Yet another approach is to microfabricate arrays of explosive microthrusters which consist of micromachined cavities filled with solid propellant. A micromachined hotplate is bonded on top of each cavity to allow ignition of the microthrusters one at a time [27][28]. Finally, silicon machining has been used to fabricate compact cold gas thrusters consisting of bonded Si chips to form a reaction chamber and compact nozzle [29]. A good overview of micropropulsion for Cubesats can be found in [30]. A Cold Gas Microthruster (CGMT) from Marotta Scientific Controls was flown on NASA's ST5 mission. More highly integrated cold-gas thrusters are expected to be flown in 2009. Formation flying missions such NASA \& ESA's Laser Interferometer Space Antenna (LISA) planned for 2019 may well use MEMSbased micro-propulsion.

\begin{tabular}{|c|c|c|}
\hline MEMS Device & Flown? (mission) & $\begin{array}{l}\text { Estimated TRL of the MEMS for } \\
\text { Space applications }\end{array}$ \\
\hline $\begin{array}{l}\text { Inertial Navigation } \\
\text { (accelerometers, gyroscopes) }\end{array}$ & Yes, almost routinely for NASA & High \\
\hline Pressure sensor & Yes, routine for launch vehicles & High \\
\hline Magnetometer & Yes (Cubesats) & High \\
\hline AFM (atomic force microscope) & In 2008 (Phoenix) & Medium- High \\
\hline Sun sensor & In 2008 (Delfi 3C) & Medium - High \\
\hline Micro-fluidics & Yes (3x Shuttle, and Genesat) & Medium \\
\hline Bolometer & In 2009 (Planck) & Medium \\
\hline Optical switching & No & Medium \\
\hline $\begin{array}{l}\text { Propulsion (ion, cold gas, colloid, solid } \\
\text { propellant) }\end{array}$ & In 2006 (ST5) & Medium \\
\hline Thermal control & In 2006 (ST5) & Medium \\
\hline RF switch and variable capacitor & In 2000 (OPAL picosats) & Low -Medium \\
\hline MEMS oscillator & No & Low - Medium \\
\hline $\begin{array}{l}\text { Adaptive optics and MOEMS-based } \\
\text { optical instruments }\end{array}$ & $\begin{array}{l}\text { No, planned for James Webb Space } \\
\text { Telescope (2013) }\end{array}$ & Low - Medium \\
\hline
\end{tabular}

Table 1: Summary of which MEMS technologies have been flown, and rough estimate of Technology Readiness Level (TRL). 


\section{CUBESATS, PICO AND NANOSATELLITES}

The generally accepted definition [32] for pico-satellites is a spacecraft with a wet mass (i.e., mass including propellant) of $1 \mathrm{~kg}$ or less, nano-satellites have mass between 1 and $10 \mathrm{~kg}$, and micro-satellites range from 10 to $100 \mathrm{~kg}$. For comparison, a typical telecom satellite in Geostationary orbit (GEO) has a mass of 1000 to $5000 \mathrm{~kg}$.

Large satellites have long development times, of order 5 to 15 years. Smaller satellites can be developed much faster. Picosatellites and Nanosatellites are natural users of MEMS because they require very high levels of integration and miniaturization in order to fit data handling, communication, attitude determination and control, payload, structure, batteries and other subsystems in such a small volume. A short overview of recent pico and nanosatellites is given below.

The Cubesat standard was defined in 1999 at the California Polytechnic State University, San Luis Obispo, CA and at Stanford University's Space Systems Development Lab, Palo Alto, CA, as a means to provide affordable access to space, initially primarily for academic institutions. Cubesats are cubic satellites $10 \mathrm{~cm}$ on a side, with a mass of up to $1 \mathrm{~kg}$. Detailed specifications can be found at [33]. Cubesats can also be built in a "triple" standard, $10 \times 10 \times 30 \mathrm{~cm}^{3}$, maximum mass of $3 \mathrm{~kg}$. Single Cubesats are typically launched in groups of three, and Cubesats are generally "piggyback" launches: the main customer of the launch vehicle chooses the orbit for its conventional-size satellites; once those main spacecraft are in orbit, the Cubesats are ejected from the launcher in a slightly different orbit to avoid any collision. There are no dedicated Cubesat launchers. Several companies offer Cubesat launch services, freeing the Cubesat builder from negotiations with the various launch providers as well as taking care of export control procedures.

There are currently approximately 80 Cubesats under development, and 40 have been launched from a variety of American, Russian, Japanese, and Indian launch vehicles. The field is active, with 5 small companies offering kits and parts specifically for the Cubesat standard [34]. While Cubesats serve in large part to teach students about system engineering and about space systems, they are also ideal vehicles for proving in or testing novel technologies that are not yet acceptable for larger commercial missions because of a lack of flight history. It is a reasonable approach to use missions which can accept large risk (such as Cubesat missions) to prove in novel technologies, or to provide the first demonstration of space application of a technology that has been shown to be reliable on Earth. The main limitation of using Cubesats or other nanosats to increase the Technical Readiness Level (TRL) of MEMS components is the generally short life (less than 1 year typically) of these small satellites, which precludes learning how a MEMS device degrades after many years in space.

For cost, lead time and availability reasons, Cubesats are generally built using commercial components, not space qualified or radiation tolerant parts. While using such parts may reduce the lifetime of Cubesats, it also means they are flying very recent technologies. The reliability of MEMS for space is discussed in [9][35] and the radiation sensitivity of MEMS to radiation is reviewed in [8]. Despite their lack of radiation shielding, radiation is generally not a major concern for Cubesats and most nanosats because they operate in low-Earth orbit, and have a lifetime probably limited by the degradation of their rechargeable batteries rather than the 3 to $15 \mathrm{krad}$ they will receive per year.

The use of MEMS in Cubesats has focused on their use in the attitude determination subsystem: inertial sensors (gyroscopes), magnetometers, and optical sensors such as sun sensors and star trackers. Magnetometers and gyroscopes are typically COTS devices, while sun sensors and star trackers are space specific developments. These are presented in the following section.

There are several nanosatellites in orbit. For example, the OPAL $13.5 \mathrm{~kg}$ satellite launched in 2000 held two picosats, each with an RF MEMS switch from Rockwell Scientific on board. The two picosats were ejected once OPAL was in orbit. One goal was to observe how MEMS devices could operate after storage in space. The picosats were turned on after one year in LEO orbit, and operated correctly [17].

MEPSI, a MEMS-based PICOSAT inspector, is a series of picosats developed by Aerospace Corp for DARPA, and have been launched in tethered pairs from the cargo bay of the Space Shuttle on flights STS -113 in 2002 and STS-116 in 2006. They have a combined mass of $3.5 \mathrm{~kg}$, and dimension of $10 \times 10 \times 12.5 \mathrm{~cm}^{3}$. The ones flown in 2006 have a 5 thruster cluster system using a MEMS pressure transducer. Figure 1 is a NASA photo showing two tethered MEPSI satellites being launched from the Shuttle cargo bay.

NASA's Space Technology 5 (ST5) was a set of three $25 \mathrm{~kg}$ spacecraft, launched in 2006, that operated as a constellation and demonstrated several new technologies, including the MEMS louvers mentioned above, and a cold gas thruster using MEMS components. 


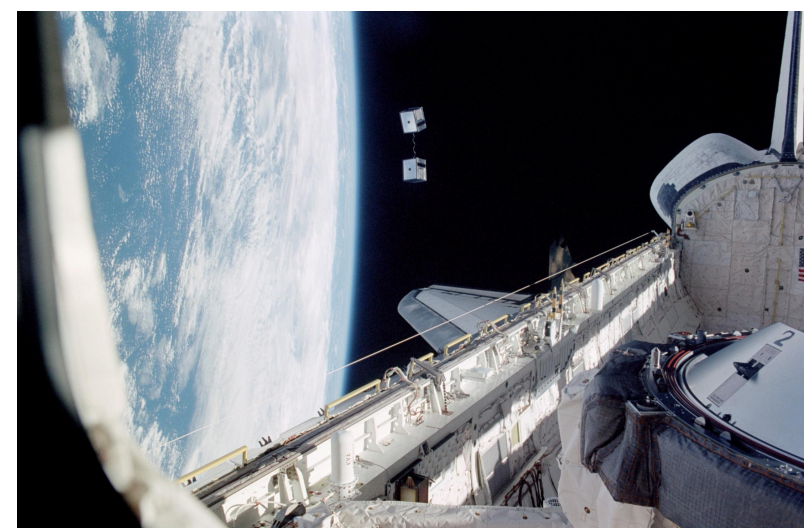

Figure 1. Two MEPSI nano-satellites just after ejection from the cargo-bay of the Space Shuttle on mission STS-116 in December 2006. Photo credit: NASA.

\section{MEMS-BASED ADCS ON CUBESATS}

The Attitude Determination and Control System (ADCS) is the subsystem where MEMS are playing the biggest role in Cubesats, because MEMS sensors offer high performance, high reliability, and low-power consumption, and because this is field where highly reliable commercial devices are available at low cost (a few dollars per axis for accelerometers, less than $\$ 100$ for gyroscopes). Propulsion (which may be part of the ADCS system if the propulsion serves to orient the spacecraft) is also an active field.

The University of Illinois's dual-cube cubesat ION (Illinois Observing NanoSatellite) included a microvacuum arc thruster ( $\mu \mathrm{VAT})$ [37]. The system consists of 4 micro-thrusters allowing two-axis rotation. Sadly ION was destroyed along with 11 other Cubesats when the launch vehicle exploded in 2006 only 86 seconds after lift-off.

Delft University in the Netherlands is planning to fly a MEMS-based cold gas thruster developed by TNO, TU Delft, and U Twente on its triple-format (3 liter volume) Cubesat Delfi-n3Xt [38], with launch planned for 2010. Here the MEMS technology (nozzles, vales) and cold-gas generation allows very important mass and volume reduction, enabling a Cubesat to have propulsion, thus greatly increasing the range of missions which Cubesats can carry out.

A good example of the use of commercial MEMS devices on a Cubesat is the Danish Cubesat AAUSAT-II, launched in 2008, carrying 6 Analog Devices Single Chip Yaw Rate Gyroscopes (model ADXRS401), and one 3-axis magnetometer model HMC1053 from Honeywell as part of the attitude determination system. The sensors operated as expected in orbit.

SwissCube [39] is a Cubesat developed in Switzerland, lead by the EPFL Space Center, with launch planned for March 2009. It will image the atmospheric airglow, which is light emitted most strongly at $762 \mathrm{~nm}$ from an oxygen layer $85 \mathrm{~km}$ to $100 \mathrm{~km}$ in altitude, as illustrated in Figure 2. To take pictures of the airglow, SwissCube must be pointed at the limb of the Earth, and requires attitude determination accurate to better than $1^{\circ}$. The only way to obtain the required performance within the mass, volume and power budgets was to use MEMS components. The goal is not to prove in the MEMS components for their own sake, but rather MEMS are unavoidable for SwissCube because high-performance attitude determination on Cubesat missions is only possible with MEMS technology.

The MEMS gyroscope selected for SwissCube was the ADXRS614 from Analog Device because of its low power consumption and a high sensitivity for low rotation rates. The gyroscopes are needed principally during eclipses (approximately $30 \%$ of each orbit is in eclipse). Three gyroscopes are mounted at $90^{\circ}$ to each other, one on the main ADCS printed circuit board, and two on smaller board mounted perpendicularly to the ADCS board, as shown in Figure 3. 
The same 3-axis Honeywell magnetometer (model HMC1053) was selected as for the AAUSAT-II mission. Knowing the position of the satellite in its orbit, and the local vector of the magnetic field allows the determination of the relative orientation of the satellite with respect to the Earth.

When SwissCube is not in the shadow of the earth, the attitude determination system is completed by another set of MEMS sensors, micromachined Sun sensors developed and fabricated at the Danish Technical University (DTU). Very similar sun sensors, also from DTU, were flown on the Danish Cubesat DTUSat1 in 2003. Unfortunately DTUSat1 did not operate in orbit for reasons that could not be determined. These sun sensors are photodiodes with micromachined slits that each provide two analog output currents, each proportional to one of the two angles between the plane of the sun sensor and the vector to the sun [40]. Six sun sensors are used, one on each face of the spacecraft, as shown in Figure 4. If the position of the sun with respect to the Earth is known (e.g. from the time and from the orbit parameters), the orientation of the satellite with respect to the Earth can be readily obtained from the angle of one satellite face to the sun vector.

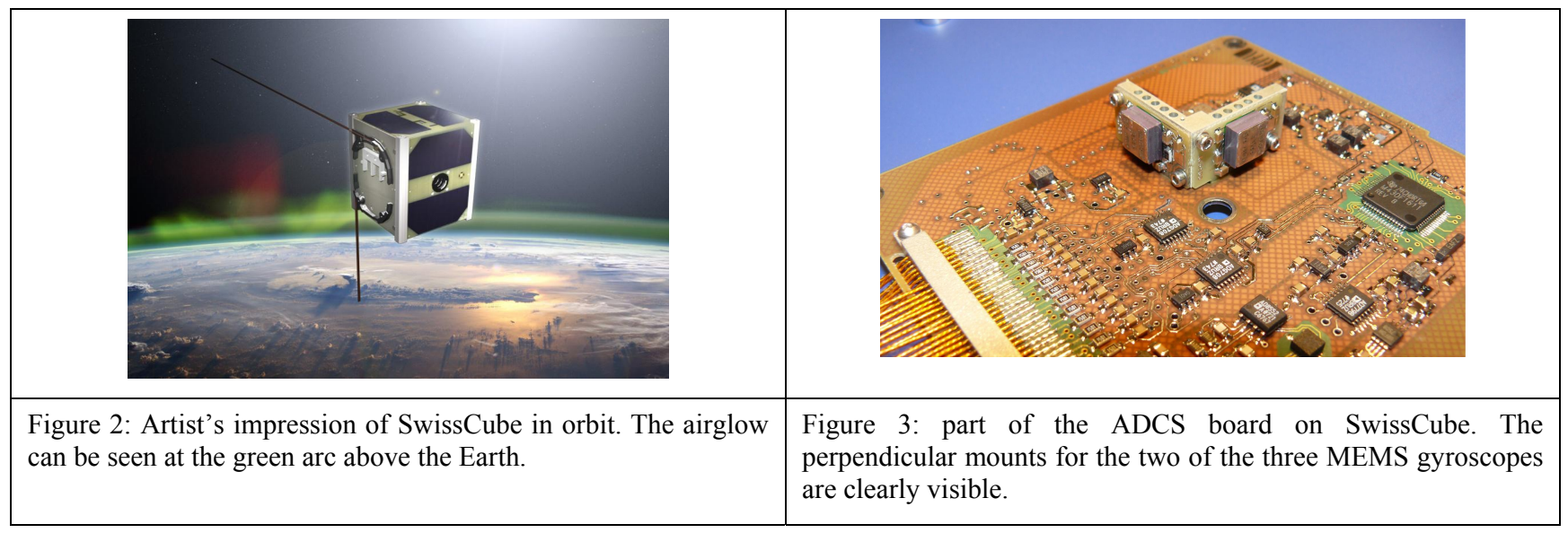

\begin{tabular}{|c|c|}
\hline 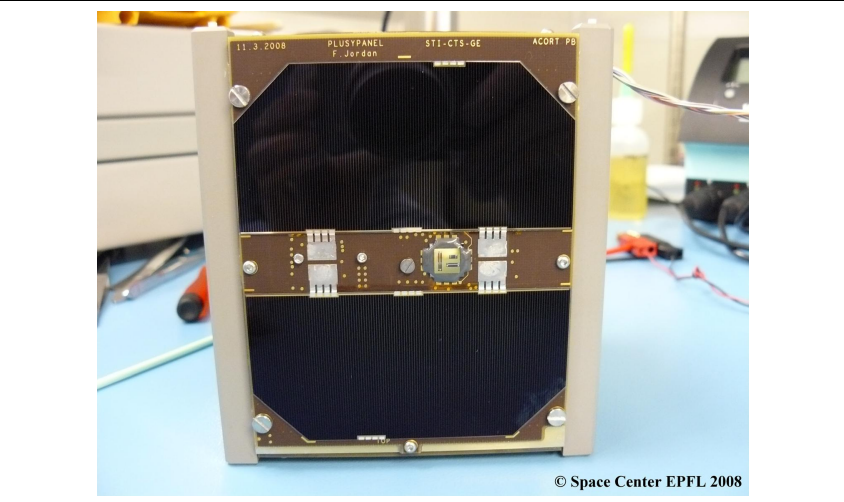 & 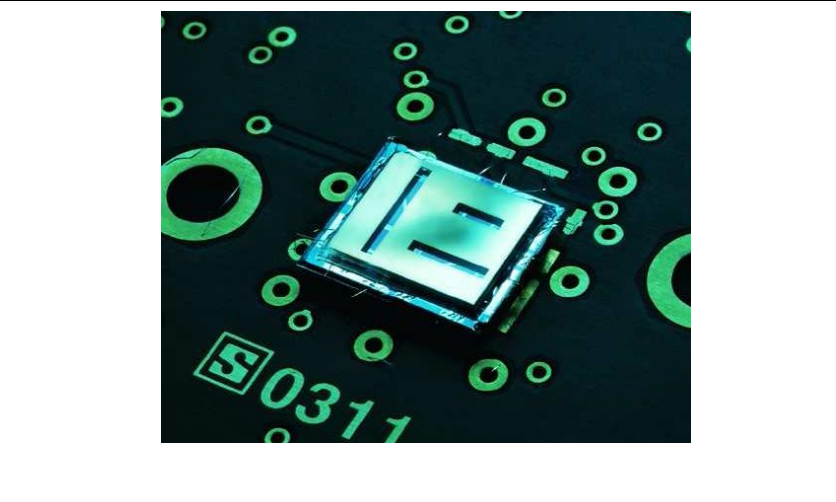 \\
\hline $\begin{array}{l}\text { Figure 4: One side of SwissCube }\left(10 \times 10 \mathrm{~cm}^{2}\right) \text {. At the center of } \\
\text { each face, between the top and bottom solar panels, the MOEMS } \\
\text { sun sensor from DTU can be seen (just to the right of the central } \\
\text { hole) }\end{array}$ & $\begin{array}{l}\text { Figure 5: Optical micrograph of one of DTU MOESM 2-axis sun } \\
\text { sensors [DTU] }\end{array}$ \\
\hline
\end{tabular}

\section{CONCLUSIONS}

MEMS technologies are increasingly used in space. They are routinely flown in Cubesats, small $1 \mathrm{~kg}$ satellites, where high levels of risk are accepted, and because operation without MEMS entails enormous performance penalties for such 
small and highly integrated systems. The attitude determination subsystem is currently the part of the pico and nanosatellites that is based on MEMS sensors. Future Cubesats will rely even more heavily on MEMS technology.

Picosatellites and nanosatellites have much smaller mass and power budgets than large multi-ton commercial satellites, and hence, along with scientific satellites, are the early adopters of MEMS in space. Scientific missions are starting to accept to MEMS components, both because of their high performance, as well as because of important mass and power savings.

MEMS qualification procedures and radiation-tolerant control electronics for MEMS are progressing quickly, and need to mature further before MEMS solutions are widely accepted in commercial spacecraft.

\section{ACKNOWLEDGEMENTS}

H.S. thanks R. Krpoun of the EPFL-LMTS, G. Röthlisberger and M. Noca of the EPFL Space Center and the entire SwissCube team for helpful discussions.

\section{REFERENCES}

[1] http://www.yole.fr/pagesAn/products/mis.asp

[2] Koons, H. C., J. E. Mazur, R. S. Selesnick, J. B. Blake, J. F. Fennell, J. L. Roeder, \& P. C. Anderson, The Impact of the Space Environment on Space Systems, Aerospace Corp. report no. TR-99(1670)-1, 20 July 1999

[3] Microengineering Aerospace Systems, H. Helvajian, ed, The Aerospace Press, 1999

[4] MEMS and Microstructures in Aerospace Applications, Robert Osiander, M. Ann Garrison Darrin, John L. Champion, eds, CRC Press 2005

[5] "MEMS in Space", S. Cass, IEEE Spectrum, p.56, July 2001

[6] Personal communication, Coumar Oudea, EADS-Astrium Space Transportation

[7] "The FAMARS Instrument: An Atomic Force Microscope for the Phoenix Mission", D. Parrat, et al., Fourth International Conference on Mars Polar Science and Exploration (2006), Abstract \#8047, http://www.lpi.usra.edu/meetings/polar2006/pdf/8047.pdf

[8] "Radiation Sensitivity of MEMS Devices", H. Shea, to appear Journal of Micro/Nanolithography, MEMS, and MOEMS, 2009

[9] B. Stark, "MEMS Reliability Assurance Guidelines for Space Applications", JPL Publication 99-1, 1999

[10] "Overview of MEMS/NEMS technology development for space applications at NASA/JPL", by T. George, Proceedings of SPIE, Proc. SPIE Int. Soc. Opt. Eng. 5116, 136 (2003)

[11] "MEMS for space applications: a reliability study", S. Barthe, F. Pressecq, L. Marchand, 4th Round Table on MNT for Space. 20-22 May 2003, ESTEC, Noordwijk, Netherlands. Available at: https://escies.org/public/mnt4/

[12] "Reliability of COTS MEMS Accelerometer Under Shock And Thermomechanical Cycling ", by Reza Ghaffarian, 2001 SMTA International Conference

[13] "Evaluation of Thermo-Mechanical Stability of COTS Dual-Axis MEMS Accelerometers for Space Applications", by A. K. Sharma and A. Teverovsky, Proceedings EEE Links Electronic Packaging and Space Parts, January 2001

[14] ECSS-Q-60-11A: Derating and end-of-life parameter drifts - EEE components, (European Cooperation for Space Standardization) available at: http://www.ecss.nl/

[15] http://genesat.arc.nasa.gov/

[16] www.radantmems.com

[17] "Microelectromechanical system radio frequency switches in a picosatellite mission", by J.Yao et al., Smart Mater. Struct. 10, p.1196-1203, 2001

[18] "Reliability of RF-MEMS", I. De Wolf et al., 4th Round Table on MNT for Space. 20-22 May 2003, ESTEC, Noordwijk, Netherlands. Available at: https://escies.org/public/mnt4/

[19] http://phoenix.lpl.arizona.edu/mission.php

[20] "Response of a MEMS Microshutter operating at $60 \mathrm{~K}$ to ionizing radiation", S. Buchner et al., IEEE Trans. on Nuclear Science, vol.54, no. 6, p 2463, 2007

[21] "Reliability Assessment and Lifetime Testing with Micro-Mirrors", S. Manhart et al., 4th Round Table on MNT for Space. 20-22 May 2003, ESTEC, Noordwijk, Netherlands. Available at: https://escies.org/public/mnt4/ 
[22] M. Beasley et al., "MEMS thermal switch for spacecraft thermal control", Proc. SPIE, Vol. 5344, 98 (2004); DOI: $10.1117 / 12.530906$

[23] "Micropropulsion for small spacecraft: a new challenge for field effect electric propulsion and microstructured liquid metal ion sources", by J. Mitterauer. Surface and Interface Analysis, Vol. 36, Issue 5-6 , p. 380 - 386 (2002)

[24] R. Krpoun et al, "Microfabrication and test of an integrated colloid thruster" 2008 Proc. 21 st IEEE Int. Conf. on Micro Electro Mechanical System (Tucson, USA) 0234 pp 964-967

[25] "Microfabricated Electrospray Thrusters for Spacecraft Propulsion", R. Krpoun, Ph.D. thesis, Ecole Polytechnique Fédérale de Lausanne, Switzerland, No. 4255 (2008).

[26] B. Gassend, "A Fully Microfabricated Two-Dimensional Electrospray Array with Applications to Space Propulsion", $\mathrm{PhD}$ thesis, Electrical Engineering and Computer Science, Massachusetts Institute of Technology, June 2007.

[27] "Digital MicroPropulsion", Lewis, et al. Sensors \& Actuators A, 2000, 80(2), p.143-154

[28] C. Rossi et al, "Matrix of $10 \times 10$ addressed solid propellant microthrusters: Review of the technologies", Sensors and Actuators A: Physical, Vol. 126, Issue 1, 2006, pp. 241-252. doi:10.1016/j.sna.2005.08.024

[29] "A hybrid cold gas microthruster system for spacecraft", J. Köhler, J. Bejhed, H. Kratz, F. Bruhn, U. Lindberg, K. Hjort, L. Stenmark, Sensors and Actuators A (Physical), A97-98:587-98, April 2002.

[30] Mueller, J.: "Thruster Options for Microspacecraft: A Review and Evaluation of Existing Hardware and Emerging Technologies", AIAA paper 97-3058, 33rd Joint Propulsion Conference and Exhibit, Seattle, WA, July 6-9, 1997

[31] "Integrated System To Analyze The Genetic Effects Of The Space Environment On Living Cells In Culture: Genesat", by A. J. Ricco1 et al., p. 527, Proceedings of the 9th International conference on miniaturized systems for Chemistry and Life Science ( TAS 2005), Boston, October 2005

${ }^{[32]}$ http://centaur.sstl.co.uk/SSHP/sshp_classify.html

[33] http://cubesat.org

[34] http://cubesat.atl.calpoly.edu/pages/suppliers.php

[35] "Reliability of MEMS for space applications", H. Shea, Proc SPIE (6111), 61110A, 2006, Photonics West 2006

[36] http://www.prismasatellites.se/?sid=902

[37] "MicroVacuum Arc Thruster Design for a Cubesat class Satellite", F. Ryasanek et al., $16^{\text {th }}$ Annula US Conference on Small Satellites, SSC02-I-2

[38] http://www.n3xt.delfispace.nl/

[39] http://swisscube.epfl.ch

[40] M. Pedersen and J.H. Hales, "Linear two-axis MOEMS Sun Sensor", M.Sc. thesis, Technical University of Denmark (DTU), 2006 